\title{
The efficacy and safety of up to 24 weeks of triptorelin therapy following conservative surgery in patients with deep infiltrating endometriosis (DIE): a multicentre, prospective, non-interventional study in China
}

\author{
Li-bo Zhu ${ }^{1}$, Zheng Guan ${ }^{2}$, Yan Huang ${ }^{3}$, Keqin Hua ${ }^{4}$, Liguo Ma ${ }^{5}$, Jian Zhang ${ }^{6}$, Dazhen \\ Yang $^{7}$, Valerie Perrot ${ }^{8}$, Hongbo $\mathrm{Li}^{9}$, and Xinmei Zhang ${ }^{1}$ \\ ${ }^{1}$ Womens Hospital, Zhejiang University School of Medicine \\ ${ }^{2}$ Chinese PLA General Hospital, Beijing, China \\ ${ }^{3}$ The Second Affiliated Hospital of Army Medical University \\ ${ }^{4}$ Obstetrics and Gynecology Hospital of Fudan University \\ ${ }^{5}$ Shenzhen People's Hospital, Guangdong Province, China \\ ${ }^{6}$ International Peace Maternity and Child Health Hospital of the China Welfare Institute, \\ Shanghai, China \\ ${ }^{7}$ Nanjing Maternity and Child Health Care Hospital, Nanjing, Jiangsu Province, China \\ ${ }^{8}$ Ipsen, Boulogne-Billancourt, France \\ ${ }^{9}$ Affiliation not available
}

May 18, 2020

\begin{abstract}
OBJECTIVE: To evaluate the efficacy and safety of up to 24 weeks of triptorelin treatment after conservative surgery for deep infiltrating endometriosis (DIE). DESIGN: Multicentre, prospective, non-interventional. SETTING: 18 tertiary hospitals in China. POPULATION: Premenopausal women aged [?] 18 years treated with triptorelin 3.75 mg once every 28 days for up to 24 weeks after conservative surgery for DIE METHODS: Endometriosis symptoms were assessed, using a visual analogue scale $(0-10 \mathrm{~cm})$ or numerical range $(0-10)$, at baseline (pre-surgery) and routine visits 3, 6, 9, 12, 18 and 24 months after surgery. MAIN OUTCOME MEASURES: Change in symptom intensity over time. RESULTS: A total of 384 women were analysed (mean [SD] age, 33.4 [6.2] years). Scores for all symptoms assessed (pelvic pain, dysmenorrhoea, ovulation pain, dyspareunia, menorrhagia, metrorrhagia and gastrointestinal and urinary symptoms) decreased from baseline over 24 months. Cumulative improvement rates in pelvic pain, dysmenorrhoea, ovulation pain and dyspareunia were $74.4 \%, 83.6 \%, 55.1 \%$ and $66.9 \%$, respectively. The 24 -month cumulative recurrence rate ([?] 1 symptom) was $22.2 \%$. The risk of symptom recurrence was higher in patients with [?] 2 versus 1 lesion (odds ratio [OR] 2.539; 95\% CI: 1.458-4.423; p = 0.001) and patients with moderate (OR 5.733; 95\% CI: 1.623-20.248; $\mathrm{p}=0.007$ ) or severe (OR 8.259; 95\% CI: $2.449-27.851 ; \mathrm{p}=0.001$ ) pain versus none/mild pain. Triptorelin was well tolerated. CONCLUSIONS: Triptorelin after conservative surgery for DIE improved symptoms over 24 months of follow up. FUNDING: Sponsored by Ipsen
\end{abstract}

Tweetable abstract (character count, 102; limit 110)

In China: Triptorelin after conservative surgery for deep infiltrating endometriosis improved symptoms

Introduction (word count, 384; limit, 400) 
Endometriosis is defined by the presence of endometrial-like tissue outside the uterus, most commonly on the peritoneal and visceral surfaces of the pelvis, which triggers a chronic inflammatory reaction. ${ }^{1}$ Women of reproductive age are predominantly affected, with the main symptoms being pain (i.e. ovulation pain, dysmenorrhoea, dyspareunia and chronic pelvic/abdominal pain) and infertility. ${ }^{1-3}$ The overall prevalence of endometriosis in women of reproductive age is estimated at $2-10 \% .,{ }^{5}$ However, the prevalence of endometriosis is $71-87 \%$ among women suffering from chronic pelvic pain and $50 \%$ among infertile women. ${ }^{6,7}$

Endometriosis can be divided into three types based on severity: superficial peritoneal endometriosis (least severe), ovarian endometriosis, and deep infiltrating endometriosis (DIE, most severe; also known as deep endometriosis, or DE) ${ }^{89}$ DIE is the most aggressive clinical form of the disease, characterized by deep infiltration and the most severe pain symptoms. Furthermore, of the three clinical forms, DIE is the most difficult to manage and is associated with high direct and indirect treatment costs. ${ }^{10}$

Surgery is often the preferred treatment in cases of DIE and the effectiveness of conservative surgery in patients with DIE has been demonstrated. ${ }^{11,} 12$ However, complete removal of endometriosis lesions during surgery cannot be guaranteed, leaving the possibility that residual lesions may continue to grow and new lesions may develop. In addition, surgery is associated with the development of adhesions within the pelvic cavity. Symptom recurrence after surgery is common, with a reported recurrence rate of $38 \%-51 \%$ within 3 years. ${ }^{13}$

A variety of medical therapies are now available for the treatment of symptomatic endometriosis. ${ }^{14}$ Gonadotropin-releasing hormone agonists (GnRH-a) have been recommended as second-line therapies for improving symptoms. ${ }^{10}$ Triptorelin is one of the most commonly used GnRH-a and has been demonstrated to improve the symptoms and fertility outcomes of patients with DIE. ${ }^{15,16}$

Although multiple studies have shown that GnRH-a treatment after surgery can improve post-operative pain and may facilitate a reduction in the rate of symptom recurrence, ${ }^{17-19}$ the effectiveness and optimal duration of GnRH-a treatment after surgery in DIE are still debated in China. This study aimed to evaluate whether up to 24 weeks of treatment with triptorelin following conservative surgery can help manage the symptoms of patients with DIE, reduce symptom recurrence rate, and improve fertility status over a 24-month follow-up period in clinical practice in China.

\section{Materials and methods}

This multi-centre, prospective, non-interventional, observational study was conducted in 18 tertiary hospitals in China between 8 September 2013 and 13 July 2018. The study was approved by the Institutional Review Board at Women's Hospital, School of Medicine, Zhejiang University and by ethical committees of all study centres and was registered at www.clinicaltrials.gov (NCT01942369).

\section{Patients}

Patients were screened using electronic diagnostic records and were eligible for inclusion if they: were premenopausal women aged [?] 18 years with a clinical diagnosis of DIE; had received surgery within one month of the study start date; had been prescribed triptorelin; and were mentally and physically able to describe their symptoms and answer questions. Exclusion criteria were: pregnant or lactating women; premenopausal women who may reach menopause within 3 years of surgery; a history of allergic reaction to triptorelin or one of the excipients; a history of treatment with other drugs within 3 months and GnRH-a therapy within 6 months prior to the study; and women who were potentially non-compliant or unsuitable for the study for other reasons, as judged by the investigator.

All included patients provided written, informed consent.

\section{Treatment and follow up}

Patients received triptorelin acetate $3.75 \mathrm{mg}$ as an intramuscular injection (Diphereline@, Ipsen) once in the cycle of 28 days for up to 24 weeks ([?] 6 injections). The prescription and number of required treatment cycles 
were determined by physicians based on routine clinical practice, without external intervention. Moreover, other treatments and management of adverse events were in accordance with routine clinical practice.

Patients were assessed at baseline (pre-surgery) and then at routine post-operative hospital visits every 3 months in the first year of follow up and every 6 months in the second year of follow up. At each visit, data and information relating to endometriosis symptoms and pregnancy status, as well as imaging and laboratory examinations were collected.

\section{Efficacy outcomes}

In the Core Outcomes in Women's and Newborn Health (CROWN) database, a core outcome set (COS) for endometriosis is currently in development. The primary outcome in this study was the change in intensity/severity of endometriosis symptoms from baseline (pre-surgery) during the 24-month follow up. Symptoms of pelvic pain, dysmenorrhoea, pain at the time of ovulation and dyspareunia were assessed using a visual analogue scale (VAS) with a range from 0 to $10 \mathrm{~cm}$, and symptoms of amenorrhea, menorrhagia, metrorrhagia and gastrointestinal and urinary discomfort were assessed using a numerical scale ranging from 0 to 10 . A score of 0 indicated that the patient was free of pain or symptoms, while a score of 10 represented pain or symptoms that were severe and intolerable. Symptoms with a VAS or numerical score $>0$ and [?] $3,>3$ and $<7$, and [?] 7 were considered as mild, moderate and severe, respectively. An improvement in symptoms was defined as a reduction of at least $3 \mathrm{~cm}$ or 3 units from pre-surgery levels. The proportions of patients with symptoms overall and with symptoms of different intensity/severity levels were also assessed.

Secondary efficacy outcomes were the rates of symptom recurrence and pregnancy. Symptom recurrence was defined as an increase of more than $3 \mathrm{~cm}$ or 3 units on the VAS or numerical score compared with the lowest previous score.

\section{Exploratory analyses}

Exploratory analyses of predictive factors for both symptom recurrence and the duration of triptorelin treatment were also conducted. For symptom recurrence, factors evaluated included age, BMI, duration of medical attention, reproductive status, comorbidities, history of surgery, surgical procedures, the site and number of DIE lesions, pain prior to surgery, pain intensity, number of triptorelin doses and concomitant medications after GnRHa. Factors assessed for their association with treatment duration included age at surgery, DIE lesion, previous surgical diagnosis of endometriosis, previous hormonal treatment for endometriosis, history of pregnancy, intensity of dyspareunia, dysmenorrhoea, menorrhagia, gastrointestinal, urinary and pain symptoms prior to surgery and infertility.

\section{Safety outcomes}

All spontaneously reported adverse events were collected up to one month after the last triptorelin injection.

\section{Statistical analysis}

Baseline characteristics were assessed with descriptive statistical analysis; the mean and standard deviation (SD) or median (range) are presented for continuous variables and frequency distribution (n, \%) presented for categorical variables. Changes in the intensity of symptoms over time are presented, as well as the proportion (\%) of patients with symptoms of different intensity/severity against time. The cumulative improvement and recurrence rates were estimated using logistic regression.

Univariate and multivariate binary logistic regression models controlling for baseline characteristics and numbers of triptorelin injections were used to assess risk factors for symptom recurrence. Univariate and multivariate Cox proportional regression models controlling for patient demographics and clinical baseline characteristics were used to identify factors associated with triptorelin treatment duration. The factors significant at $20 \%(p<0.20)$ in the univariate analysis were entered in the multivariate model. All tests were two sided and a level of $p<0.05$ was considered significant. All statistical analysis was performed in SAS version 9.21 (SAS Institute, Cary, NC, USA). 


\section{Patient and public involvement}

There was no patient or public involvement in this study.

\section{Funding}

This study was sponsored by Ipsen.

\section{Results}

\section{Patients}

In total, 402 patients were screened for the study. Of these, 399 were enrolled and 384 (96.2\%) were included in the final analysis. Fifteen patients were excluded from the final analysis because of missing data on triptorelin administration.

The baseline characteristics of patients are shown in Table 1. The mean +- SD age of patients was 33.4 +- 6.2 years; $80(20.8 \%)$ patients had received a previous surgical diagnosis of endometriosis, $4(5.0 \%)$ of them had received previous surgery for DIE. Overall, 63 (16.4\%) patients had received a previous medical treatment for endometriosis.

During surgery, 149 (38.8\%) patients displayed peritoneal superficial endometriosis and 357 (93.0\%) patients had endometrioma. DIE lesions were located on the intestine, bilateral uterosacral ligament, vagina, ureter and bladder in $55.5 \%, 50.5 \%, 9.6 \%, 2.6 \%$ and $2.1 \%$ of patients, respectively. The majority of patients $(64.8 \%)$ had a history of pregnancy; of these, $33.7 \%$ had a history of miscarriage, $32.5 \%$ of voluntary abortion, $14.1 \%$ of therapeutic abortion and $3.2 \%$ of ectopic abortions.

A total of 35 (9.1\%) patients received 1-2 injections of triptorelin, 191 (49.7\%) patients received 3-5 injections, and $158(41.2 \%)$ patients received 6 injections. The mean duration of drug exposure was 13.0 weeks (range: 0 to 29 weeks).

\section{Symptoms}

Changes in the intensity of symptoms over time from baseline (pre-surgery) to 24 months after surgery are summarized in Figure 1. The scores for all symptoms decreased during follow up. At baseline and 24 months, respectively, symptom scores [mean (SD)] were: pelvic pain, 2.8 (3.0) and 0.3 (0.8); dysmenorrhoea, 5.0 (3.4) and 0.5 (1.2); ovulation pain, $1.2(2.1)$ and 0.1 (0.7); dyspareunia, $1.6(2.4)$ and $0.1(0.4)$; gastrointestinal symptoms, 1.5 (2.7) and $0.1(0.6)$.

At baseline, the proportions of women reporting pelvic pain, dysmenorrhoea, ovulation pain, dyspareunia or gastrointestinal symptoms were $58.1 \%$ (223/384), $84.1 \%$ (323/384), 35.9\% (138/384), $42.4 \%(163 / 384)$ and $31.4 \%$ (119/379), respectively; at 24 months, these proportions were reduced to $16.0 \%(34 / 213), 23.0 \%$ (49/213), 6.6\% (14/213), 5.6\% (12/213) and 2.8\% (6/213), respectively (Figure 2). From baseline to the end of follow up, there were considerable reductions in the proportions of patients with severe pelvic pain (15.1\% to $0.5 \%$ ), dysmenorrhoea ( $41.4 \%$ to $0.5 \%$ ), ovulation pain ( $4.4 \%$ to $0.5 \%)$, dyspareunia $(6.0 \%$ to $0 \%$ ) and gastrointestinal symptoms $(9.2 \%$ to $0 \%)$. Similarly, the proportions of patients with moderate symptoms were reduced from baseline over the 24 months for pelvic pain $(21.9 \%$ to $0.5 \%)$, dysmenorrhoea $(21.4 \%$ to $3.8 \%$ ), ovulation pain $(8.9 \%$ to $0 \%$ ), dyspareunia (14.8\% to $0.5 \%)$ and gastrointestinal symptoms $(8.4 \%$ to $0.9 \%)$.

For menorrhagia, metrorrhagia and urinary symptoms, there were fewer reported cases at baseline $(10.7 \%$, $9.6 \%$ and $7.6 \%$, respectively) compared with the other symptoms. However, reductions in the proportions of patients with these symptoms from baseline to month 24 of follow up were still observed (Figure 2).

Among patients with a symptom intensity score $>0$ at baseline, the cumulative rates of improvement in endometriosis symptoms at 12 months were $72.6 \%$ for pelvic pain, $83.3 \%$ for dysmenorrhoea, $55.1 \%$ for ovulatory pain, $66.9 \%$ for dyspareunia, $78.0 \%$ for menorrhagia, $59.5 \%$ for metrorrhagia, $75.6 \%$ for gastrointestinal 
symptoms and $58.6 \%$ for urinary symptoms. Almost all improvements occurred in the first 12 months. Similar cumulative improvement rates were observed for all symptoms at 24 months (Table 2).

\section{Symptom recurrence and pregnancy}

The 24-month cumulative rate of symptom recurrence, defined as [?] 1 symptom of recurrence, was $22.2 \%$ (Table 2). The 12-month and 24-month cumulative recurrence rates, respectively, were $3.1 \%$ and $4.4 \%$ for pelvic pain, $10.0 \%$ and $16.9 \%$ for dysmenorrhoea, $1.3 \%$ and $1.9 \%$ for ovulatory pain, $0.6 \%$ and $1.3 \%$ for dyspareunia, $0.9 \%$ and $1.6 \%$ for menorrhagia, $0 \%$ and $0 \%$ for metrorrhagia, $1.3 \%$ and $3.1 \%$ for gastrointestinal symptoms and $0 \%$ and $0 \%$ for urinary symptoms.

In total, 59 patients were diagnosed as infertile. Among these patients, the 12-month and 24-month cumulative rates of pregnancy were $18.6 \%$ and $27.1 \%$, respectively; of the pregnancies at 24 months, $62.5 \%$ were spontaneous pregnancies and $37.5 \%$ were pregnancies arising from assisted reproductive technology (Table 2).

\section{Predictive factors for symptom recurrence}

The univariate logistic regression analysis identified DIE lesion in the intestine or in uterosacral ligament, pre-surgery pain symptoms with or without GI symptoms and with or without infertility, number of DIE lesion locations, intensity of pain and number of doses of triptorelin as the most significant $(p<0.20)$ risk factors for symptom recurrence (Table S1). In the multivariate logistic regression model, the risk of symptom recurrence was significantly higher in patients with two or more lesions compared with those with one lesion (OR 2.539; 95\% CI: 1.458-4.423; $p=0.001$ ) and in patients with moderate pain (OR 5.733; 95\% CI: $1.623-20.248 ; p=0.007$ ) or severe pain (OR 8.259;95\% CI: 2.449-27.851; $p=0.001$ ) compared with those with none or mild pain.

\section{Predictive factors for duration of triptorelin treatment}

The univariate Cox regression analysis identified age, associated DIE lesion, history of hormonal treatment for endometriosis, pre-surgery intensity of dysmenorrhoea, ovulation pain and gastrointestinal symptoms and infertility as the most significant $(p<0.20)$ predictive factors for triptorelin treatment duration (Table S2). In a multivariate Cox regression analysis, the duration of triptorelin treatment was longer in patients older versus younger at the time of surgery (HR $0.983 ; 95 \%$ CI: $0.968-1.000 ; p=0.044$ ), who previously received hormonal treatment for endometriosis compared with those who did not (HR 0.724; 95\% CI:0.543-0.950; $p$ $=0.024$ ), and shorter in patients who were infertile versus fertile (HR 1.401; 95\% CI:1.046-1.844; $p=0.019$ ).

\section{Safety outcomes}

Overall, 135 (35.2\%) adverse events were reported (Table S3). Menopausal symptoms, including hot flushes and sweat/night sweat, were the most common related adverse events, with an incidence of $18.0 \%(\mathrm{n}=69)$, followed by muscle/bone pain, agrypnia, and dizziness with incidences of $5.5 \%(\mathrm{n}=21), 2.9 \%(\mathrm{n}=11)$ and $1.3 \%(\mathrm{n}=5)$, respectively. There were no deaths or adverse events leading to withdrawals from the study.

Discussion (word count, 1169; limit, 1200)

\section{Main findings}

In this multicentre, prospective, non-interventional study in China, treatment with triptorelin $3.75 \mathrm{mg}$ once every 28 days for up to 24 weeks after conservative surgery in patients with DIE improved pelvic pain, dysmenorrhoea, pain at the time of ovulation, dyspareunia, menorrhagia, metrorrhagia, gastrointestinal symptoms, and urinary symptoms from baseline over the 24-month follow-up period. In addition, the proportions of patients with these symptoms decreased over time, particularly those with moderate and severe symptoms. The cumulative rates of improvement in individual symptoms over the 24-month period ranged from $55.1 \%$ to $83.6 \%$.

The rate of symptom recurrence was low; at 24 months, the overall rate of recurrence was $22.2 \%$, but for all individual symptoms except dysmenorrhoea, the recurrence rates were less than $5 \%$. 
The exploratory analyses of risk factors indicated that patients with more lesions and more intense pain may have a higher risk of recurrence, and that older age at surgery, fertility and a history of hormonal treatment are predictive factors for a longer duration of triptorelin treatment.

The administration of triptorelin was well tolerated, with no serious treatment-related adverse events. Perimenopausal symptoms were the most common adverse effects.

\section{Strengths and limitations}

The key strength of this study is that it is the first real-world study to focus on the efficacy and safety of triptorelin treatment after surgery in patients with DIE in multiple centres in China. These data reflect current clinical practice in China and may have implications for future clinical guidance. Additionally, the study analysed risk factors for recurrence, which may inform clinical decisions regarding prevention of recurrence.

There are, however, several limitations. As a non-interventional study conducted in routine clinical practice, missing data are inevitable. Also, in the absence of a control group, the efficacy and safety results can only be compared with published studies. In addition, the treatment regimen and duration were adjustable, according to routine clinical practice, which may have influenced the outcomes. Furthermore, because the first follow-up visit was 3 months after surgery (owing to practical clinical considerations), it was difficult to determine the immediate effectiveness of surgery and compare with follow-up assessments. Importantly, DIE has a complex phenotype and, as such, evaluation of symptom improvement alone may not capture the effects of treatment on all aspects of the disease.

\section{Interpretation}

At present, there is no consensus regarding the treatment regimen for DIE, although a stepwise approach is usually suggested. ${ }^{14}$ Surgery is a common treatment option for patients with DIE. ${ }^{20}$ However, whether surgery is the best therapeutic option strongly depends on the experience of the attending surgeon and the probability of being able to achieve total excision of DIE foci. ${ }^{10}$ A previous report suggested that conservative surgery should be offered if the total excision of DIE foci is possible. ${ }^{10}$ Regarding the alleviation of pain, the benefits of surgery should always be balanced with the risk of intraoperative complications. ${ }^{21,}{ }^{22}$ For infertile women with DIE, whether to opt for in vitro fertilization (IVF) treatment or surgery first can be a dilemma. Pregnancy complications, such as placenta previa, gestational hypertonia, and intrauterine growth restriction have been shown to be more common in women with DIE even after complete excision compared with women without endometriosis. ${ }^{23,} 24$

The improvements in pelvic pain, dysmenorrhoea, pain at the time of ovulation, dyspareunia, menorrhagia, metrorrhagia, gastrointestinal symptoms, and urinary symptoms with triptorelin treatment in this study are consistent with findings from previous studies on the improvement in pain symptoms with triptorelin, ${ }^{15,} 16,25$ and provide further support for the use of triptorelin after surgery for DIE.

Owing to inconsistencies in the definition of recurrence and the length of follow up, comparing results between studies on endometriosis is difficult. In the present study, recurrence was evaluated based on symptoms of endometriosis. The overall 12-month cumulative rate of symptom recurrence was $12.8 \%$, which is higher than the symptom and sign recurrence rate of $5 \%$ reported after surgery and two months of treatment with triptorelin over a follow up of at least 12 months after restoration of a regular cycle in a randomized study. ${ }^{26}$ However, the 24 -month cumulative rate of symptom recurrence of $22.2 \%$ is similar to the endometrioma relapse rate of $21 \%$ reported in a randomized controlled trial with 5 -year follow up. ${ }^{27}$ The 12 -month and 24-month recurrence rates of individual symptoms were less than $5 \%$, with the exception of those for dysmenorrhoea ( $10.0 \%$ and $16.9 \%$, respectively), which were similar to the previously reported 6 -month dysmenorrhoea recurrence rate of $13.3 \%$ following completion of 24 weeks of triptorelin treatment. ${ }^{28}$ Differences in study design and patient inclusion and exclusion criteria are likely to contribute to these differences in symptom recurrence rates. 
The rate of symptom recurrence gradually increased over time, highlighting the importance of long-term healthcare in symptom management.

Identifying risk factors for recurrence may assist early intervention and prevention. In addition to the multiple DIE locations and more severe pain symptoms identified as risk factors for recurrence in the present study, a positive family history has been shown to be a risk factor for endometrioma recurrence. ${ }^{13}$ Particular care and attention are required when managing these high-risk patients.

The overall 12-month and 24-month pregnancy rates in this study were $10.9 \%$ and $17.2 \%$, respectively. For the subpopulation of infertile patients, pregnancy rates were higher, at $18.6 \%$ at 12 months and $27.1 \%$ at 24 months. This 12-month pregnancy rate for infertile patients was slightly lower than that reported for infertile women with endometriosis treated with triptorelin and followed up for up to 12 months after restoration of a regular cycle in a randomized controlled trial $(27.5 \%) .{ }^{26}$ However, as the study did not directly capture how many women tried to become pregnant during the 24 months follow-up, the interpretation of this observation is unclear. Furthermore, the present 24-month pregnancy rate for infertile patients was substantially lower than the 24-month pregnancy rate of $80 \%$ observed in infertile patients treated with triptorelin in the randomized controlled trial by Xue et al. ${ }^{19}$ A possible reason for this difference is the older age of patients in this study compared with the study by Xue et al. (mean age, 33.4 vs. 26.7 years), as the problem of infertility is greater and the planning of pregnancy less common in older patients.

A recent randomized trial by Angoni et al. demonstrated improvements in pelvic pain in patients with DIE with GnRH-a treatment, in agreement with the present results, and also showed that the effect of GnRH-a was closely related to the duration of treatment. ${ }^{18}$ In this study, the duration of triptorelin treatment showed great variation across the study population. Most women received either three $(30.0 \%)$ or six injections (39.6\%). The Cox-regression model suggested that patients who were older at the time of surgery, fertile or with history of hormonal treatment for endometriosis tended to be treated with triptorelin for longer. Thus, indicating that treatment duration is associated with patient health status, disease severity and history of therapy.

The most common adverse effects in patients treated with triptorelin were menopause symptoms (hot flash/flush, sweat/night sweat) in agreement with results from previous studies. ${ }^{15,16,19,29}$

\section{Conclusion}

This multicentre, prospective and non-interventional study demonstrated that treatment with triptorelin for up to 24 weeks following conservative surgery for DIE had a beneficial impact on pelvic pain, dysmenorrhoea, ovulation pain and dyspareunia. Over 24 months, the recurrence rate of symptoms was low and triptorelin was generally well tolerated.

\section{Acknowledgements}

The authors thank all patients involved in the study, as well as their caregivers, care team, investigators and research staff in participating institutions. The authors also thank Beijing Preintell Biomed Co., Ltd, who provided medical writing support, which was sponsored by Ipsen, in accordance with Good Publication Practice guidelines. Furthermore, the authors gratefully acknowledge all the investigators of research institutions for their contribution to the study: Li Hong (Remin Hospital of Wuhan University), Juxin Zhang (Henan Provincial People's Hospital), Min Hao (The Second Hospital of Shanxi University), Jianliu Wang (People's Hospital of Peking University), Ouping Huang (Jiangxi Maternal and Child Health Hospital), Hongbo Wang (Wuhan Union Hospital of Tongji Medical College of Huazhong University of Science and Technology), Jun Zhang (Beijing Anzhen Hospital of Capital Medical University), Dong Zhao (The First Maternal and Infant Hospital of Shanghai), Weidong Zhao (Anhui Provincial Cancer Hospital), Zhiqing Liang (Southwest Hospital of the Third Military Medical University), Lifang Sun (The Fourth Medical College of Peking University and Jishuitan Orthopaedic College of Tsinghua University).

\section{Disclosures of interest}


Valerie Perrot and Hongbo Li are employees of Ipsen. The other authors have stated explicitly that there are no conflicts in connection with the article.

\section{Contribution to authorship}

LZ performed the study and was a major contributor in writing the manuscript. ZG, YH, KH, LM,JZ and DY performed the study and made a contribution to the study design and manuscript draft. VP and HL analysed and interpreted clinical data, provided feedback on the manuscript. XZ designed and performed the study, was a major contributor in writing the manuscript and gave final approval of the version to be submitted. All authors read and approved the final manuscript.

\section{Details of ethics approvals}

The study was approved by the Institutional Review Board at Women's Hospital, School of Medicine, Zhejiang University and by ethical committees of all study centres in the date of June 24, 2013 with the reference number of 2013 (0017).

\section{Funding}

Sponsorship, article processing charges and the open access fee for this study were funded by Ipsen.

\section{Data Sharing}

Where patient data can be anonymised, Ipsen will share all individual participant data that underlie the results reported in this article with qualified researchers who provide a valid research question. Study documents, such as the study protocol and clinical study report, are not always available. Proposals should be submitted to DataSharing@Ipsen.com and will be assessed by a scientific review board. Data are available beginning 6 months and ending 5 years after publication; after this time, only raw data may be available.

\section{References}

1. Kennedy S, Bergqvist A, Chapron C, D'Hooghe T, Dunselman G, Greb R, et al. ESHRE guideline for the diagnosis and treatment of endometriosis. Hum Reprod. 2005 Oct;20(10):2698-704.

2. LaMonica R, Pinto J, Luciano D, Lyapis A, Luciano A. Incidence of Septate Uterus in Reproductive-Aged Women With and Without Endometriosis. J Minim Invasive Gynecol. 2016 May-Jun;23(4):610-3.

3. Morassutto C, Monasta L, Ricci G, Barbone F, Ronfani L. Incidence and Estimated Prevalence of Endometriosis and Adenomyosis in Northeast Italy: A Data Linkage Study. PLoS One. 2016;11(4):e0154227.

4. Dunselman GA, Vermeulen N, Becker C, Calhaz-Jorge C, D'Hooghe T, De Bie B, et al. ESHRE guideline: management of women with endometriosis. Hum Reprod. 2014 Mar;29(3):400-12.

5. Eskenazi B, Warner ML. Epidemiology of endometriosis. Obstet Gynecol Clin North Am. 1997 Jun;24(2):235-58.

6. Luciano DE, Luciano AA. Management of endometriosis-related pain: an update. Womens Health (Lond). 2011 Sep;7(5):585-90.

7. Meuleman C, Vandenabeele B, Fieuws S, Spiessens C, Timmerman D, D'Hooghe T. High prevalence of endometriosis in infertile women with normal ovulation and normospermic partners. Fertil Steril. 2009 Jul;92(1):68-74.

8. Santulli P, Chouzenoux S, Fiorese M, Marcellin L, Lemarechal H, Millischer AE, et al. Protein oxidative stress markers in peritoneal fluids of women with deep infiltrating endometriosis are increased. Hum Reprod. 2015 Jan;30(1):49-60.

9. Working group of Esge E, Wes, Keckstein J, Becker CM, Canis M, Feki A, et al. Recommendations for the surgical treatment of endometriosis. Part 2: deep endometriosis. Hum Reprod Open. 2020;2020(1):hoaa002. 
10. Szubert M, Zietara M, Suzin J. Conservative treatment of deep infiltrating endometriosis: review of existing options. Gynecol Endocrinol. 2018 Jan;34(1):10-4.

11. Hudelist G, Aas-Eng MK, Birsan T, Berger F, Sevelda U, Kirchner L, et al. Pain and fertility outcomes of nerve-sparing, full-thickness disk or segmental bowel resection for deep infiltrating endometriosis-A prospective cohort study. Acta Obstet Gynecol Scand. 2018 Dec;97(12):1438-46.

12. Uccella S, Gisone B, Serati M, Biasoli S, Marconi N, Angeretti G, et al. Functional outcomes of nervesparing laparoscopic eradication of deep infiltrating endometriosis: a prospective analysis using validated questionnaires. Arch Gynecol Obstet. 2018 Sep;298(3):639-47.

13. Campo S, Campo V, Gambadauro P. Is a positive family history of endometriosis a risk factor for endometrioma recurrence after laparoscopic surgery? Reprod Sci. 2014 Apr;21(4):526-31.

14. Vercellini P, Buggio L, Frattaruolo MP, Borghi A, Dridi D, Somigliana E. Medical treatment of endometriosis-related pain. Best Pract Res Clin Obstet Gynaecol. 2018 Aug;51:68-91.

15. Bergqvist A, Bergh T, Hogstrom L, Mattsson S, Nordenskjold F, Rasmussen C. Effects of triptorelin versus placebo on the symptoms of endometriosis. Fertil Steril. 1998 Apr;69(4):702-8.

16. Choktanasiri W, Boonkasemsanti W, Sittisomwong T, Kunathikom S, Suksompong S, Udomsubpayakul U, et al. Long-acting triptorelin for the treatment of endometriosis. Int J Gynaecol Obstet. 1996 Sep;54(3):237-43.

17. Jee BC, Lee JY, Suh CS, Kim SH, Choi YM, Moon SY. Impact of GnRH agonist treatment on recurrence of ovarian endometriomas after conservative laparoscopic surgery. Fertil Steril. 2009 Jan;91(1):40-5.

18. Angioni S, Pontis A, Dessole M, Surico D, De Cicco Nardone C, Melis I. Pain control and quality of life after laparoscopic en-block resection of deep infiltrating endometriosis (DIE) vs. incomplete surgical treatment with or without GnRHa administration after surgery. Arch Gynecol Obstet. 2015 Feb;291(2):36370.

19. Xue H, Liu M, Hao W, Li Y. Clinical evaluation of laparoscopic surgery combined with triptorelin acetate in patients with endometriosis and infertility. Pak J Med Sci. 2018 Sep-Oct;34(5):1064-9.

20. Roman H, group F. A national snapshot of the surgical management of deep infiltrating endometriosis of the rectum and colon in France in 2015: A multicenter series of 1135 cases. J Gynecol Obstet Hum Reprod. 2017 Feb;46(2):159-65.

21. Ferrero S, Alessandri F, Racca A, Leone Roberti Maggiore U. Treatment of pain associated with deep endometriosis: alternatives and evidence. Fertil Steril. 2015 Oct;104(4):771-92.

22. Brown J, Farquhar C. An overview of treatments for endometriosis. JAMA. 2015 Jan 20;313(3):296-7.

23. Nirgianakis K, Gasparri ML, Radan AP, Villiger A, McKinnon B, Mosimann B, et al. Obstetric complications after laparoscopic excision of posterior deep infiltrating endometriosis: a case-control study. Fertil Steril. 2018 Aug;110(3):459-66.

24. Darwish B, Stochino-Loi E, Pasquier G, Dugardin F, Defortescu G, Abo C, et al. Surgical Outcomes of Urinary Tract Deep Infiltrating Endometriosis. J Minim Invasive Gynecol. 2017 Sep - Oct;24(6):998-1006.

25. Ferrero S, Venturini PL, Gillott DJ, Remorgida V. Letrozole and norethisterone acetate versus letrozole and triptorelin in the treatment of endometriosis related pain symptoms: a randomized controlled trial. Reprod Biol Endocrinol. 2011 Jun 21;9:88.

26. Alborzi S, Hamedi B, Omidvar A, Dehbashi S, Alborzi S, Alborzi M. A comparison of the effect of short-term aromatase inhibitor (letrozole) and GnRH agonist (triptorelin) versus case control on pregnancy rate and symptom and sign recurrence after laparoscopic treatment of endometriosis. Arch Gynecol Obstet. 2011 Jul;284(1):105-10. 
27. Loverro G, Carriero C, Rossi AC, Putignano G, Nicolardi V, Selvaggi L. A randomized study comparing triptorelin or expectant management following conservative laparoscopic surgery for symptomatic stage IIIIV endometriosis. Eur J Obstet Gynecol Reprod Biol. 2008 Feb;136(2):194-8.

28. Kang JL, Wang XX, Nie ML, Huang XH. Efficacy of gonadotropin-releasing hormone agonist and an extended-interval dosing regimen in the treatment of patients with adenomyosis and endometriosis. Gynecol Obstet Invest. 2010;69(2):73-7.

29. Michel Cosson MD, Denis Querleu, M.D., Jacques Donnez, M.D., Patrick Madelenat, M.D., Philippe Koninckx, M.D., Alain Audebert, M.D., and Hubert Manhes, M.D. Dienogest is as effective as triptorelin in the treatment of endometriosis after laparoscopic surgery: results of a prospective, multicenter, randomized study. FERTILITY AND STERILITY. 2002;77(4):9.

\section{Table and Figure captions}

Table 1. Baseline demographics and clinical characteristics of study population

Table 2. Efficacy outcomes in patients treated with triptorelin for up to 24 weeks following conservative surgery for DIE

\section{Figure 1. Changes in the intensity of endometriosis symptoms over time}

A visual analogue scale (VAS) or a numerical scale was used to evaluate pain or other symptoms, respectively. A score of 0 indicated that the patient was free of pain or other symptoms while 10 indicated the pain or symptoms were severe and intolerable. Mean scores and standard deviations of symptom intensities against time are presented.

Figure 2. Changes in the proportions of patients with different intensities of endometriosis symptoms over time

Cumulative proportion of patients with different symptom intensities are shown against time. A VAS or numerical score [?] 7 was considered as severe, a score of $>3$ and $<7$ as moderate, and a score of $>0$ and [?] 3 as mild.

\section{Table 1.}

\begin{tabular}{ll}
\hline Characteristics & Total $(\mathbf{N = 3 8 4})$ \\
\hline Age $($ years), mean $\pm \mathrm{SD}$ & $33.4 \pm 6.2$ \\
BMI $\left(\mathrm{kg} / \mathrm{m}^{2}\right)$, mean $\pm \mathrm{SD}$ & $21.0 \pm 2.8$ \\
Age when first medical attention sought (years) $(\mathrm{n}=327)$, mean $\pm \mathrm{SD}$ & $31.4 \pm 6.3$ \\
Age at first endometriosis surgical diagnosis (years) $(\mathrm{n}=80)$, mean $\pm \mathrm{SD}$ & $29.8 \pm 5.3$ \\
Location of DIE lesions, n (\%) & \\
Intestine & $213(55.5)$ \\
Vagina & $37(9.6)$ \\
Ureter & $10(2.6)$ \\
Bladder & $8(2.1)$ \\
Left uterosacral ligament & $42(10.9)$ \\
Right uterosacral ligament & $31(8.1)$ \\
Bilateral uterosacral ligament & $194(50.5)$ \\
Treatment history & \\
Previously surgically diagnosed with endometriosis, $\mathrm{n}(\%)$ & $80(20.8)$ \\
Previous surgery for endometriosis (other than DIE), $\mathrm{n}(\%)$ & $43(53.8)$ \\
Previous surgery for DIE, $\mathrm{n}(\%)$ & $4(5.0)$ \\
Previous medical treatment for endometriosis, $\mathrm{n}(\%)$ & $63(16.4)$ \\
Oral contraceptive pills treatment duration (months) (n=10), mean $\pm \mathrm{SD}$ & $15.2 \pm 24.4$
\end{tabular}




\begin{tabular}{ll}
\hline Characteristics & Total $(\mathbf{N}=\mathbf{3 8 4})$ \\
\hline GnRH-a treatment duration (months) $(\mathrm{n}=18)$, mean $\pm \mathrm{SD}$ & $3.9 \pm 1.9$ \\
Progesterone treatment duration (months) $(\mathrm{n}=5)$, mean $\pm \mathrm{SD}$ & $27.6 \pm 25.9$ \\
Traditional Chinese medication treatment duration (months) $(\mathrm{n}=38)[$ mean $\pm \mathrm{SD}]$ & $10.6 \pm 15.2$ \\
Reproductive history, $\mathrm{n}(\%)$ & \\
Previous pregnancy history & $249(64.8)$ \\
Previous miscarriage history* & $84(33.7)$ \\
Previous voluntary abortion history* & $81(32.5)$ \\
Previous therapeutic abortion history* & $35(14.1)$ \\
Previous ectopic abortions* & $8(3.2)$ \\
\hline
\end{tabular}

BMI, body mass index; DIE, deep infiltrating endometriosis; GnRH-a, gonadotropin-releasing hormone agonist

*The proportions were calculated based on pregnant women.

Table 2.

\begin{tabular}{|c|c|c|c|c|}
\hline Outcomes & 12 months & 12 months & 24 months & 24 months \\
\hline & $\mathbf{N}$ & n (\%) & $\mathbf{N}$ & n (\%) \\
\hline \multicolumn{5}{|l|}{$\begin{array}{l}\text { Cumulative } \\
\text { improvement } \\
\text { rate }\end{array}$} \\
\hline Pelvic pain & 223 & $162(72.6)$ & 223 & $166(74.4)$ \\
\hline Dysmenorrhoea & 323 & $269(83.3)$ & 323 & $270(83.6)$ \\
\hline $\begin{array}{l}\text { Pain at time of } \\
\text { ovulation }\end{array}$ & 138 & $76(55.1)$ & 138 & $76(55.1)$ \\
\hline Dyspareunia & 163 & $109(66.9)$ & 163 & $109(66.9)$ \\
\hline Menorrhagia & 41 & $32(78.0)$ & 41 & $32(78.0)$ \\
\hline Metrorrhagia & 37 & $22(59.5)$ & 37 & $22(59.5)$ \\
\hline \multicolumn{2}{|c|}{ Gastrointestinal symptdrin 9} & $90(75.6)$ & 119 & $90(75.6)$ \\
\hline Urinary symptoms & 29 & $17(58.6)$ & 29 & $17(58.6)$ \\
\hline $\begin{array}{l}\text { Cumulative } \\
\text { recurrence rate }\end{array}$ & 320 & $41(12.8)$ & 320 & $71(22.2)$ \\
\hline Pelvic pain & 320 & $10(3.1)$ & 320 & $14(4.4)$ \\
\hline Dysmenorrhoea & 320 & $32(10.0)$ & 320 & $54(16.9)$ \\
\hline $\begin{array}{l}\text { Pain at time of } \\
\text { ovulation }\end{array}$ & 320 & $4(1.3)$ & 320 & $6(1.9)$ \\
\hline Dyspareunia & 320 & $2(0.6)$ & 320 & $4(1.3)$ \\
\hline Menorrhagia & 320 & $3(0.9)$ & 320 & $5(1.6)$ \\
\hline Metrorrhagia & 320 & $0(0)$ & 320 & $0(0)$ \\
\hline \multicolumn{2}{|c|}{ Gastrointestinal sympto̊ß } & $4(1.3)$ & 320 & $10(3.1)$ \\
\hline Urinary symptoms & 320 & $0(0.0)$ & 320 & $0(0.0)$ \\
\hline \multicolumn{5}{|l|}{$\begin{array}{l}\text { rate, study } \\
\text { population }\end{array}$} \\
\hline $\begin{array}{l}\text { Pregnancy } \\
\text { rate, infertile } \\
\text { patients }\end{array}$ & 59 & $11(18.6)$ & 59 & $16(27.1)$ \\
\hline $\begin{array}{l}\text { Spontaneous } \\
\text { pregnancy }\end{array}$ & 11 & $8(72.7)$ & 16 & $10(62.5)$ \\
\hline
\end{tabular}




\begin{tabular}{lllll}
\hline Outcomes & $\mathbf{1 2}$ months & $\mathbf{1 2}$ months & $\mathbf{2 4}$ months & 24 months \\
\hline Assistance fertility & 11 & $3(27.3)$ & 16 & $6(37.5)$ \\
\hline
\end{tabular}

Figure 1
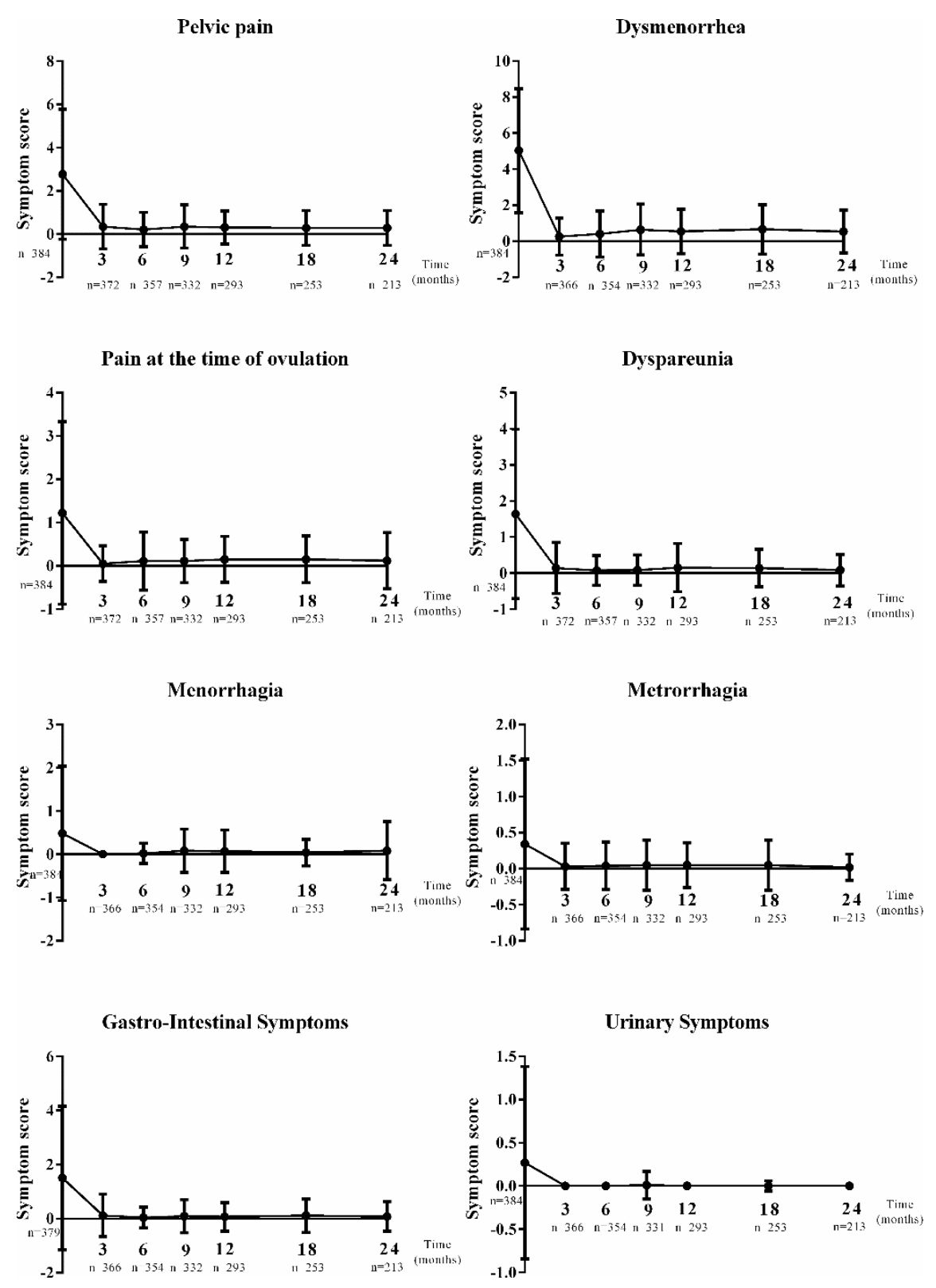

Figure 2 
Pelvic pain

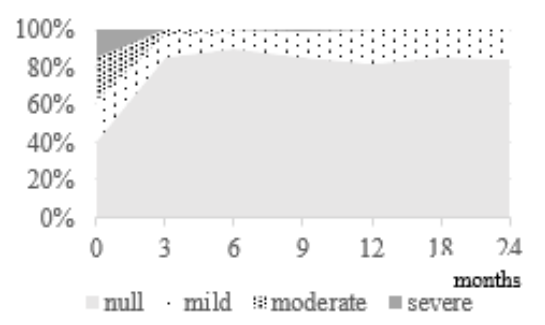

Pain at time of ovulation

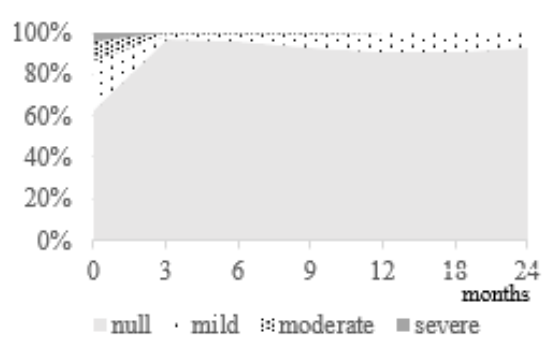

Menorrhagia

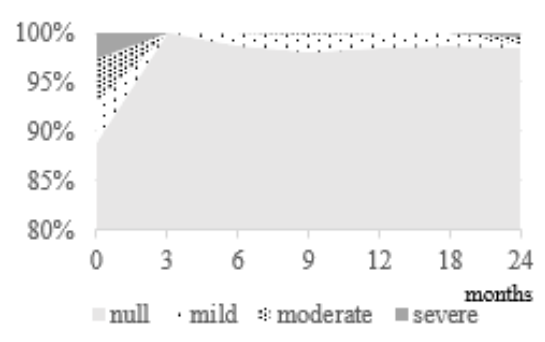

Gastro-intestinal symptoms

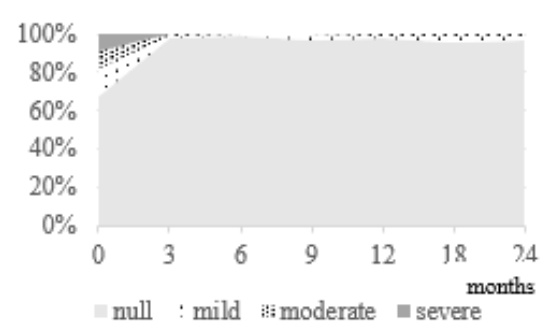

Dysmenorrhoea

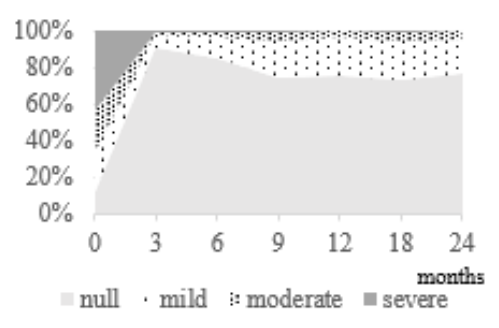

Dyspareunia
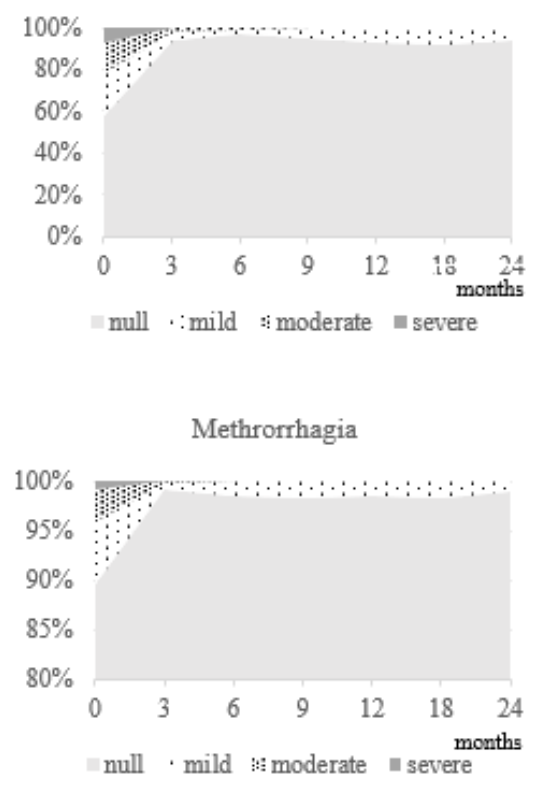

Urinary symptoms

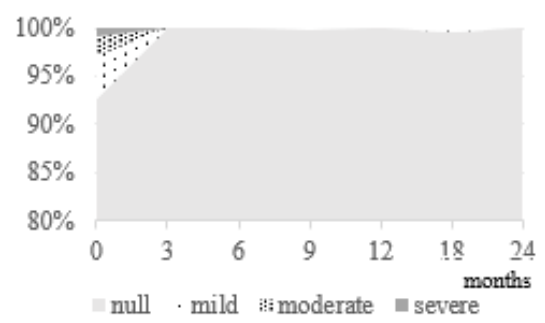

